\title{
Clinical and Radiological Results of Oxford Phase-3 Medial Unicompartmental Knee Arthroplasty
}

Sinan Karaca ${ }^{1}$, Mehmet N. Erdem ${ }^{2,3}$, Ahmet Oztermeli ${ }^{4}$, Emre Bal ${ }^{5}$, Abdullah Gogus ${ }^{6}$, Azmi Hamzaoglu 6

1. Orthopaedics and Traumatology, Sehit Prof. Dr. Ilhan Varank Sancaktepe Education and Research Hospital, Istanbul, TUR 2. Orthopaedics and Traumatology, Isik University, Istanbul, TUR 3. Orthopaedics and Traumatology, Hisar Intercontinental Hospital, Istanbul, TUR 4. Orthopaedics and Traumatology, Gebze Fatih Government Hospital, Izmit, TUR 5. Orthopaedics and Traumatology, Uskudar Goverment Hospital, Istanbul, TUR 6. Orthopaedics and Traumatology, Istanbul Florence Nightingale Hospital, Istanbul, TUR

Corresponding author: Sinan Karaca, mdsnn@hotmail.com

\section{Abstract \\ Purpose}

The aim of this retrospective study was to investigate the effectiveness of medial unicompartmental knee arthroplasty (UKA) by showing the results of the radiological and clinical outcomes of the patients.

\section{Materials and methods}

Seventy-two knees of 54 patients who underwent UKA between September 2005 and March 2011 for medial knee arthritis with a minimum follow-up of six months were evaluated. Range of motion (ROM), Hospital for Special Surgery (HSS) knee score, Knee Society Score (KSS), and Oxford Knee Score (OKS) were investigated both preoperatively and postoperatively. On the other hand, Oxford radiographic evaluation criteria were used to evaluate prostheses radiologically at the final follow-up.

\section{Results}

The average age was 53.4 years ( 47 to 79 years). The average follow-up time was 39.8 months ( 8 to 72 months). There was a significant difference between preoperative and postoperative ROM, HSS, and OKS $(p<0.05)$. Radiologically, there was no sign of arthritis on the unoperated side of the knee or failure of prosthesis detected. Before the operation, the average clinical KSS was 63.2 and improved to 91.4 after the operation. In addition, the average functional KSS was 54.9 before the operation and improved to 86.5 after the operation. The average knee flexion degree was 109.1 before the operation and there was an improvement to 123.6 degrees after the operation. Before the operation, the average HSS score was 67.5 (range, 52 to 75 ) and improved to 89.9 (range, 85 to 100) at the final control examination.

Received 06/11/2019

Review began 07/18/2019 Review ended 11/03/2019 Published 11/04/2019

(c) Copyright 2019

Karaca et al. This is an open access article distributed under the terms of the Creative Commons Attribution License CC-BY 3.0., which permits unrestricted use, distribution, and reproduction in any medium, provided the original author and source are credited.

\section{Conclusion}

This study supports the use of Oxford Phase 3 UKA, which has excellent clinical and radiological results in patients with medial knee arthritis.

Categories: Orthopedics

Keywords: knee, unicompartmental knee arthroplasty, osteoarthritis of knee, arthroplasty

\section{Introduction}

The incidence of symptomatic knee osteoarthritis is currently increasing all over the world with the aging of the population [1-3]. The treatment options of this patient population with knee osteoarthritis are debatable. Only limited pain relief and functional improvement can be achieved with the non-operative treatment options [4]. Due to this reason, arthroplasty surgery at the knee is assumed to be popular and by 2030, there will be a linear increase at the rate of $673 \%$ [5]. Unicompartmental knee arthroplasty (UKA), high tibial osteotomy (HTO), and conventional total knee arthroplasty (TKA) are the primary surgical methods of treating isolated lateral or anteromedial compartment knee arthritis [6]. OA has a multifactorial etiology, which occurs due to an interplay between systemic and local factors. Osteoarthritis is a disease that can be seen especially in the elderly population. The etiology of osteoarthritis is linked to several responsible genes. Trauma at the knee, high body mass index (BMI), family history, age, diabetes, synovitis, systemic inflammatory mediators, lower limb alignment, joint shape and dysplasia, and inflammatory diseases are causes of osteoarthritis. There is a 3.86 times increased risk of $\mathrm{OA}$ at a knee joint with a previous injury [7]. Surgical techniques and instruments are improving currently. There are some studies showing excellent results after UKA, including a decreased angle of deformity, reduced knee pain, and achieving the physiological knee motion range. In addition, these studies have shown that clinical and functional scores 
increased significantly [8-9].

Shorter incisions and decreased soft tissue damage are the advantages of UKA and there is more bone stock conserved in UKA than in TKA by providing better kinesiology and faster recovery to the patients [10-12].

The aim of this study was to investigate the clinical and radiological results of Oxford medial UKA due to medial compartment arthrosis.

\section{Materials And Methods}

This is a retrospective investigation of patients who underwent UKA. After the operation, there was a routine control physical and radiological examination. The first control was in the sixth week. The second was in the sixth month. After these controls, in the first and second-year, the final follow-up control was done.

Between September 2005 and March 2011, the 72 consecutive knees of 54 patients who had a UKA operation (Oxford Partial Knee, Biomet Orthopedics, Warsaw, Indiana), with no loss to follow-up, were enrolled in the study. Every participant signed an informed consent form.

Clinically, knee pain resistant to conservative therapy on the anteromedial side was the indication for the operation. On the other hand, radiologically, grade 4 arthritis was the indication for the operation. The exclusion criteria were joint inflammation, ruptured anterior cruciate ligament, and a history of knee surgery. Age and weight were not considered exclusion criteria.

All patients had a routine physical examination. Also, all patients had a radiological examination, including anteroposterior (AP) standing upright, patella tangential, lateral at $20^{\circ}$ of flexion, and varus/valgus stress views of both knees, which were the routine radiograph imaging (Figure 1).

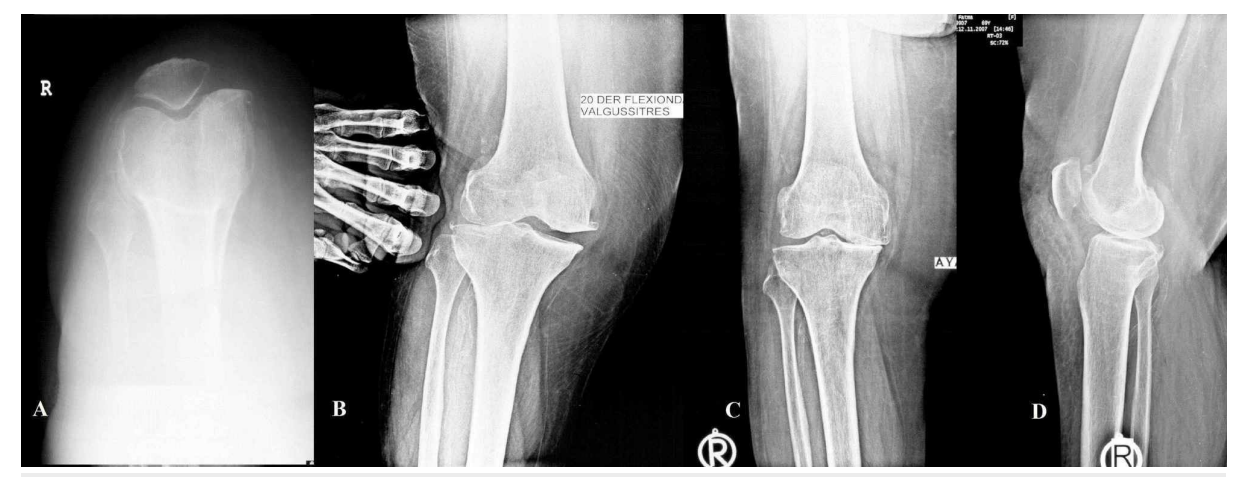

FIGURE 1: Preoperative radiological images

A. Patella tangential, B. Valgus stress, C. AP standing upright, D. Lateral at $20^{\circ}$ of flexion views

Before the operation, the knee range of motion and knee scores are taken from all patients. At the follow-up examinations, knee scores and range of motion were analyzed and noted from all patients. Implants were assessed with the Oxford radiologic evaluation criteria.

There was no ligament injury, including anterior and posterior cruciate ligaments, in any patient. At the physical examination, if an injury to the ligament is suspected, magnetic resonance imaging (MRI) was done. In some cases before the knee arthroplasty operation, a diagnostic knee arthroscopy operation was done if needed. All the patients were operated by the same senior author (AG) at Bilim University's orthopedic department between the years 2005 and 2011. In all operations, the same implant was used (Oxford Phase III).

Full weight-bearing was allowed immediately postoperatively. Intravenous first-generation cephalosporin (single-shot 2 g cefazolin) was administered preoperatively one hour before surgery. For thrombosis, prophylaxis anticoagulation therapy consisted of low molecular weight heparin (Fundoparinux 2500 IU,) which was prescribed for 21 days.

Statistical analysis was achieved using Statistical Package for the Social Sciences (SPSS) version 22 (IBM Corp, Armonk, New York). Data were studied by using a paired t-test before and after the operation. A pvalue of $<0.05$ was considered statistically significant.

\section{Results}

Changes in knee scores during the follow-up period in patients. At last follow-up control, we had two cases 


\section{Cureus}

exitus. Exitus was not related to the UKA procedure. The rest of the knees $(n=72)$ were examined at an average follow-up time of 39.8 months. The sexual orientation proportion was $47 \mathrm{females} / \mathrm{seven}$ males. Individually, 11 patients were in the 46-55 age group, 25 patients were in the 56-65 age group, and 18 patients were in the 66-79 age group. The mean age was 53.4 (47-79). The average BMI was $26.78 \mathrm{~kg} / \mathrm{m}^{2}$ $\left(22.47-31.74 \mathrm{~kg} / \mathrm{m}^{2}\right)$ (Table 1$)$.

DEMOGRAPHIC

N

All patients had a full range of motion both actively and passively on the seventh day and in the second month after the operation.

Before the operation, the average KSS was 55.11 (range, 43-68), and it increased to 96.71 (range, 88-100) after the operation. Before the operation, the functional KSS was 52.76 (range, 41-67) and it improved to 89.62 (range, 81-99) ( $\mathrm{p}<0.05$ ). Intercalarily, the HSS score improved from 53.21 (range, 38-69) to 95,74 (range, 86-100) $(\mathrm{p}<0.05)$. Before the operation, the average ROM was 120.38 degrees (range, 102-130), and it rose to 129.02 degrees (range, 115-140) after the UKA operation ( $p<0.05)$. Before the operation, the average Oxford Knee Score was 14.18 (range, 5-22) and it rose to 43.64 (range, 38-48) after the UKA operation $(\mathrm{p}<0.05)($ Table 2$)$.

\begin{tabular}{|c|c|c|c|}
\hline & Preoperative & Postoperative & $P$ values \\
\hline Oxford knee score & $14.18 \pm 4,7$ & $43,64 \pm 5,0$ & 0.024 \\
\hline HSS knee score & $53.21 \pm 9,5$ & $95,74 \pm 5,3^{*}$ & 0.038 \\
\hline KSS clinical knee score & $55.11 \pm 8,7$ & $96.71 \pm 4,3$ & $<0.001$ \\
\hline KSS functional knee score & $52.76 \pm 8,7$ & $89.62 \pm 5,1$ & $<0.001$ \\
\hline Knee ROM & $120,38 \pm 5,2$ & $129,02 \pm 4,8$ & 0.046 \\
\hline
\end{tabular}

\section{TABLE 2: Results of the knee scores at the patients who had UKA surgery}

Bone cracks around the implant, disengagement, any infectious disease, or any complications were not seen during the subsequent period.

The weight-bearing radiograph was taken preoperatively and at the last follow-up. The mean femorotibial angle evaluated, and it was $2.8^{\circ}$ varus (range, $8.7^{\circ}$ varus to $2.1^{\circ}$ valgus) before the operation and $2.8^{\circ}$ valgus (range, $2.4^{\circ}$ varus to $7.4^{\circ}$ valgus) after the operation at the final examination. Osteolysis and $\geqslant 2 \mathrm{~mm}$ abnormal radiolucency around the femoral or tibial part were not observed in any patients on radiographs.

Despite the fact that patellofemoral joint inflammation was suspected in two patients, no treatment was done. Dynamic horizontal compartment joint pain was seen in three patients, however, none of them was treated with TKA.

Even though patellofemoral joint arthritis was suspected in two patients, no treatment was required in light of the fact that they were asymptomatic. Progressive lateral compartment arthritis was observed in three patients but none of them were treated with TKA.

\section{Discussion}

One compartment arthritis is a challenging disease with the absence of high-quality treatment. However, 
there are some treatment options like TKA, UKA, and HTO. Over the years, the most preferred procedure of orthopedic surgeons was TKA. In a study, TKA and UKA were compared, and it is found that patients with UKA have reduced operation time and greater knee range of motion. In another study, it is shown that the activity levels in patients operated with UKA were better than in patients with TKA [13-15]. Since the patellofemoral compartment is preserved, the failure of the UKA can easily be revised to TKA. A study comparing primary TKA and TKA after failed UKA showed similar clinical results [16]. In our study, we found similar satisfactory results with UKA.

In a study, it is found that the clinical results of $\mathrm{HTO}$ and UKA are similar when a convenient patient selection was achieved. [17]. But as compared to HTO, UKA has increasing popularity over the years. There are points of interest like prompt weight-bearing and shorter recovery time after UKA. On the other hand, there are restrictions on weight-bearing and a longer rehabilitation period with HTO. With UKA, this process may be easier for patients, and thus UKA could be preferred. On the other hand, with reduced hospitalization time, rehabilitation period, and return to work time, UKA is more cost-effective than HTO.

In our study, functional recovery was achieved at the one-year follow-up and after that, no significant progression was observed. The literature contains clinical results that are similar to our study [18].

Patient selection is important for achieving good clinical outcomes in UKA surgery. UKA is one of the best choices of surgery for selected patients. However, many complications have been published in studies over the years [19-21].

In a study that shows perfect outcomes, it has been shown that convenient patient selection is the most important factor to achieve successful results [22]. We also use the same criteria for patient selection in our study. Due to this convenient patient selection criteria, we had similar, successful clinical and radiological results that are compatible with the literature.

In orthopedic surgery, less invasive techniques are gaining popularity because of faster recovery time and allowing for a more active lifestyle, therefore, UKA is a good surgical option for the treatment of knee osteoarthritis. Additionally, the Oxford UKA was found to be ideally appropriate for use as an outpatient procedure that lowers costs and has higher patient and surgeon satisfaction.

The main limitations of our present study are a relatively short minimum follow-up, the lack of a control group (e.g. HTO or TKA), and that the data were analyzed retrospectively.

\section{Conclusions}

Oxford mobile-bearing UKA demonstrated excellent clinical improvement and survivorship in patients. Further prospective, randomized follow-up studies should be performed to determine the long-term efficacy of the procedure.

\section{Additional Information \\ Disclosures}

Human subjects: Consent was obtained by all participants in this study. Animal subjects: All authors have confirmed that this study did not involve animal subjects or tissue. Conflicts of interest: In compliance with the ICMJE uniform disclosure form, all authors declare the following: Payment/services info: All authors have declared that no financial support was received from any organization for the submitted work. Financial relationships: All authors have declared that they have no financial relationships at present or within the previous three years with any organizations that might have an interest in the submitted work. Other relationships: All authors have declared that there are no other relationships or activities that could appear to have influenced the submitted work.

\section{References}

1. Goodfellow JW, Tibrewal SB, Sherman KP, O'Connor JJ: Unicompartmental Oxford meniscal knee arthroplasty. J Arthroplasty. 1987, 2:1-9. 10.1016/S0883-5403(87)80025-6

2. Inglis GS: Unicompartmental arthroplasty of the knee: a follow-up of 3 to 9 years . J Bone Joint Surg Br. 1984, 66:4. 10.1302/0301-620X.66B5.6501360

3. Knutson K, Jonsson G, Andersen JL, Larusdottir H, Lidgren L: Deformation and loosening of the tibial component in knee arthroplasty with unicompartmental endoprostheses. Acta Orthop Scand. 1981, 52:667673. 10.3109/17453678108992165

4. Crawford DC, Miller LE, Block JE: Conservative management of symptomatic knee osteoarthritis: a flawed strategy?. Orthop Rev. 2013, 5:2. 10.4081/or.2013.e2

5. Kurtz S, Ong K, Lau E, Mowat F, Halpern M: Projections of primary and revision hip and knee arthroplasty in the United States from 2005 to 2030. J Bone Joint Surg Am. 2007, 89:780-785.

6. Argenson JN, Parratte S, Bertani A, et al.: The new arthritic patient and arthroplasty treatment options. J Bone Joint Surg Am. 2009, 91:43-44. 10.2106/JBJS.I.00406

7. Chahla J, Piuzzi NS, Mitchell JJ, Dean C, Pascual-Garrido C, LaPrade R, Muschler G: Intra-articular cellular 
therapy for osteoarthritis and focal cartilage defects of the knee: A systematic review of the literature and study quality analysis. J Bone Joint Surg Am. 2016, 98:1511-1521. 10.2106/JBJS.15.01495

8. Pietschmann MF, Wohlleb L, Weber P, et al.: Sports activities after medial unicompartmental knee arthroplasty Oxford III-what can we expect?. Int Orthop. 2013, 37:31-37. 10.1007/s00264-012-1710-7

9. Newman JH, Ackroyd CE, Shah NA: Unicompartmental or total knee replacement? Five-year results of a prospective, randomised trial of 102 osteoarthritic knees with unicompartmental arthritis. J Bone Joint Surg Br. 1998, 80:862-865. 10.1302/0301-620X.80B5.0800862

10. Argenson JN, Chevrol-Benkeddache Y, Aubaniac JM: Modern unicompartmental knee arthroplasty with cement: a three to ten-year follow-up study. J Bone Joint Surg Am. 2002, 84:2235-2239.

11. Argenson JN, Flecher X: Minimally invasive unicompartmental knee arthroplasty. Knee. 2004, 11:341-347. 10.1016/j.knee.2003.12.002

12. Barnes CL, Mesko JW, Teeny SM, York SC: Treatment of medial compartment arthritis of the knee: a survey of the American Association of Hip and Knee Surgeons. J Arthroplasty. 2006, 21:950-956. 10.1016/j.arth.2006.01.003

13. Berger RA, Meneghini RM, Jacobs JJ, Sheinkop MB, Della Valle CJ, Rosenberg AG: Results of unicompartmental knee arthroplasty at a minimum of ten years of follow-up. J Bone Joint Surg Am. 2005, 87:999-1006. 10.2106/JBJS.C.00568

14. O'Rourke MR, Gardner JJ, Callaghan JJ, Liu SS, Goetz DD, Vittetoe DA: The John Insall Award: unicompartmental knee replacement: a minimum twenty-one-year followup, end-result study. Clin Orthop Relat Res. 2005, 440:27-37.

15. Argenson JN, Komistek RD, Aubaniac JM, Dennis DA, Northcut EJ, Anderson DT: In vivo determination of knee kinematics for subjects implanted with a unicompartmental arthroplasty. J Arthroplasty. 2002, 17:1049-1054. 10.1054/arth.2002.34527

16. Levine WN, Ozuna RM, Scott RD, Thornhill TS: Conversion of failed modern unicompartmental arthroplasty to total knee arthroplasty. J Arthroplasty. 1996, 11:797-801. 10.1016/S0883-5403(96)80179-3

17. Nwachukwu BU, McCormick FM, Schairer WW, Frank RM, Provencher MT, Roche MW: Unicompartmental knee arthroplasty versus high tibial osteotomy: United States practice patterns for the surgical treatment of unicompartmental arthritis. J Arthroplasty. 2014, 29:1586-1589. 10.1016/j.arth.2014.04.002

18. Pandit H, Jenkins C, Beard DJ, et al.: Cementless Oxford unicompartmental kneereplacement shows reduced radiolucency at one year. J Bone Joint Surg Br. 2009, 91:185-189. 10.1302/0301-620X.91B2.21413

19. Cameron HU, Jung YB: A comparison of unicompartmental knee replacement with total knee replacement . Orthop Rev. 1988, 17:983-988.

20. Stempin R, Stempin K, Kaczmarek W: Medium-term outcome of cementless, mobile-bearing, unicompartmental knee arthroplasty. Ann Transl Med. 2019, 3:41. 10.21037/atm.2018.12.50

21. Fornell S, Prada E, Barrena P, García-Mendoza A, Borrego E, Domecq G: Mid-term outcomes of mobilebearing lateral unicompartmental knee arthroplasty. Knee. 2018, 25:1206-1213. 10.1016/j.knee.2018.05.016

22. Berger RA, Meneghini RM, Jacobs JJ, et al.: Results of unicompartmental knee arthroplasty at a minimum of ten years of follow-up. J Bone Joint Surg Am. 2005, 87:999-1006. 10.2106/JBJS.C.00568 